\title{
Two-Dimensional Evaluation of Turbulent Boundary Layer Pressure Fluctuations at Cruise Flight Conditions
}

\author{
Stefan Haxter* and Carsten Spehr ${ }^{\dagger}$ \\ German Aerospace Center DLR, D-37073 Göttingen, Germany
}

\begin{abstract}
A distributed pressure transducer array was installed on an Airbus model A320 airplane and measurements were performed under various cruise flight conditions. Turbulent boundary layer pressure fluctuations were recorded at several flight altitudes and velocities. The array was installed directly in front of the wing root by replacing three pax windows with aluminum dummy windows equipped with piezo-resistive pressure sensors. Evaluating the signals via a frequency-domain array processing technique provided information about local flow phenomena. Narrow-band convectional direction was extracted from the measurement data and decay of coherence was determined for the predominant flow direction. Two dimensional coherence of turbulent structures and wavenumber spectra were compared with the empirical models of Corcos and Efimtsov.
\end{abstract}

\section{Nomenclature}

$\begin{array}{ll}A, B & \text { Corcos' coherence factors } \\ b & \text { dirty map } \\ c_{f} & \text { friction coefficient } \\ c & \text { speed of sound, } \mathrm{ms}^{-1} \\ C & \text { point spread function matrix } \\ e & \text { steering vector } \\ f & \text { frequency, Hz } \\ G & \text { cross correlation } \\ h & \text { singular value / eigenvalue } \\ \mathrm{j} & \text { imaginary unit; } \mathrm{j}=\sqrt{-1} \\ k & \text { wavenumber in scanning grid, } \mathrm{m}^{-1} \\ k_{0} & \text { wavenumber at the speed of sound, } \mathrm{m}^{-1} \\ l & \text { coherence length } \\ L & \text { exponential factor in coherence fit } \\ M a & \text { Mach number } \\ N & \text { number of input elements to } \\ & \text { singular value decomposition } \\ p & \text { pressure, Pa } \\ P & \text { wavenumber spectrum } \\ q & \text { source map } \\ r & \text { ratio of longitudinal to lateral coherence } \\ R & \text { cross spectral density matrix } \\ \Re & \text { specific gas constant of air } \\ t & \text { time, s }\end{array}$

$T \quad$ temperature, $\mathrm{K}$

$u_{c} \quad$ convective velocity

$u_{0}$ free-stream velocity

$u_{\tau} \quad$ wall shear rate

$x \quad$ aircraft longitudinal direction, $\mathrm{m}$

$y \quad$ aircraft lateral direction, $\mathrm{m}$

$z \quad$ aircraft vertical direction, $\mathrm{m}$

$\alpha \quad$ angle of maximum coherence

$\Omega \quad$ Corcos' correlation length

$\Lambda \quad$ Efimtsov' correlation length

$\Gamma \quad$ coherence

$\delta \quad$ boundary layer thickness, $\mathrm{m}$

$\rho$ density of air, $\mathrm{kg} / \mathrm{m}^{3}$

$\sigma \quad$ standard deviation

$\kappa \quad$ isentropic exponent

$\lambda \quad$ eigenvalue of SVD analysis

$\tau \quad$ wall shear stress, $\mathrm{N} / \mathrm{m}^{2}$

$\xi \quad$ longitudinal spacing, $\mathrm{m}$

$\eta \quad$ lateral spacing, $\mathrm{m}$

$\omega$ angular frequency, $\mathrm{s}^{-1}$

$\nu \quad$ kinematic viscosity of air, $\mathrm{m}^{2} \mathrm{~s}^{-1}$

Re Reynolds Number $\mathrm{u}_{\infty} \mathrm{x} / \nu$

Sh Strouhal Number $\omega \xi / \mathrm{u}_{\tau}$

\footnotetext{
*PhD student, Institute of Aerodynamics and Flow Technology - Experimental Methods, stefan.haxter@dlr.de, no member

${ }^{\dagger}$ Research Engineer, Institute of Aerodynamics and Flow Technology - Experimental Methods, carsten.spehr@dlr.de, no member
} 


\begin{tabular}{|c|c|c|}
\hline \multicolumn{3}{|r|}{ Abbreviations } \\
\hline$m, n$ & transducer index & $\mathrm{a} / \mathrm{c}$ \\
\hline$i$ & index of singular values & $F L$ \\
\hline $\max$ & maximum & PSF \\
\hline c & convective & SVD \\
\hline corc & using the Corcos model & TBL \\
\hline efim & using the Efimtsov model & WFS \\
\hline thresh & threshold value & \\
\hline 1,2 & different spacing area used & \\
\hline
\end{tabular}

Aircraft
Flight Level
Point Spread Function
Singular Value Decomposition
Turbulent Boundary Layer
Wavenumber-Frequency-Spectrum

\section{Introduction}

Passenger comfort depends to a great extent on in-flight interior aircraft noise level. ${ }^{1}$ Cabin noise sources can roughly be divided to result from turbulent boundary layer (TBL) noise, jet engine noise, and system noise, e.g. hydraulics and air conditioning. One approach to predict TBL-induced noise is to calculate the response of fuselage hull panels to excitement by turbulent boundary layer pressure fluctuations (Graham, ${ }^{2}$ $\mathrm{Liu}^{3}$ ). These fluctuations have been measured in the past in wind tunnels (Willmarth and Wooldridge, ${ }^{4}$ Ehrenfried and $\mathrm{Koop}^{5}$ ) and under free-flight conditions (Bhat, ${ }^{6}$ Efimtsov, ${ }^{7}$ Palumbo $^{8,9}$ ). In the flight tests however, there was either no reference to the alignment of the probe array to flow direction (Efimtsov, ${ }^{7}$ Palumbo ${ }^{8,9}$ ) or the possible misalignment was mentioned but not measured $\left(\right.$ Bhat $\left.^{6}\right)$. The data base for the analysis by Palumbo ${ }^{8,9}$ was provided by Rizzi ${ }^{10,11}$ who recorded the surface flow direction in the second of the two conducted flight tests. Mismatching flow direction and array alignment causes uncertainty in the determination of boundary-layer fluctuation models as mentioned by Rackl. ${ }^{12}$ The investigation presented in this paper utilizes a distributed pressure transducer array. The concept of transducer positioning on the array was comparable with that used by Ehrenfried and Koop ${ }^{5}$ in their measurement. In the present paper, array analysis provides information about the convective flow direction. Data from in-flight measurements under cruise conditions conducted by Spehr et al. ${ }^{13}$ are utilized to investigate the convectional properties of the boundary layer pressure fluctuations, including its direction. In the first part, a comparison of experimentally obtained coherence with the TBL surface pressure fluctuation models of $\operatorname{Corcos}^{14}$ and Efimtsov ${ }^{7}$ is presented.

In the second part, wavenumber spectra computed from the array measurements are presented. In the past, this computation had been performed by Arguillat ${ }^{15}$ in a wind tunnel at low speeds up to $40 \mathrm{~m} / \mathrm{s}$. In the experiments, a rotating disk equipped with transducers was used to provide different spacings for the measurement. The spectra have also been generated by Ehrenfried and Koop ${ }^{5}$ with their irregularly-spaced array in a wind tunnel. In their evaluation, a deconvolution procedure as described by the same authors ${ }^{16}$ was applied to the data to improve the resolution of the array.

\section{A. Conduction of the Flight Test}

For the in-flight measurements of pressure fluctuations underneath a turbulent boundary layer, the Advanced Technology Research Aircraft (ATRA) of the German Aerospace Center (DLR) was used as a test carrier. The aircraft was equipped with three dummy windows at a longitudinal position of $x=12.56 \mathrm{~m}$ shown in figure 1. A total of 30 Kulite pressure sensors were arranged in an optimized pattern throughout the whole available three-window area in a way that provided various different spacings between sensors in both aircraft lateral and longitudinal directions (figure 2). Each sensor was attached to the window by means of a mounting adapter and connected to the outer air with a $0.3 \mathrm{~mm}$ bearing of $4 \mathrm{~mm}$ length. The Helmholtz resonant frequency of this bearing was determined to be approximately $5 \mathrm{kHz}$ under flight conditions.

Kulite pressure probes XCL-093 were used in the array. A leadless design made the transducers more resistant to environmental corrosion. On the reference pressure side, the Kulites were attached to a reference pressure transducer (SETRA ASM1-16P) connected to an additional bearing on the center dummy window. The reference pressure system served as a low-pass filter providing a non-fluctuating pressure on the transducer reference pressure side for all the measurement points. Pressure signals were recorded using a DEWETRON DEWE-818 data acquisition system with DEWETRON DEWE-51-PCI-128 A/D-frontends at a sampling rate of $50 \mathrm{kHz}$. For further information on the flight test, refer to Spehr et al. ${ }^{13}$ 


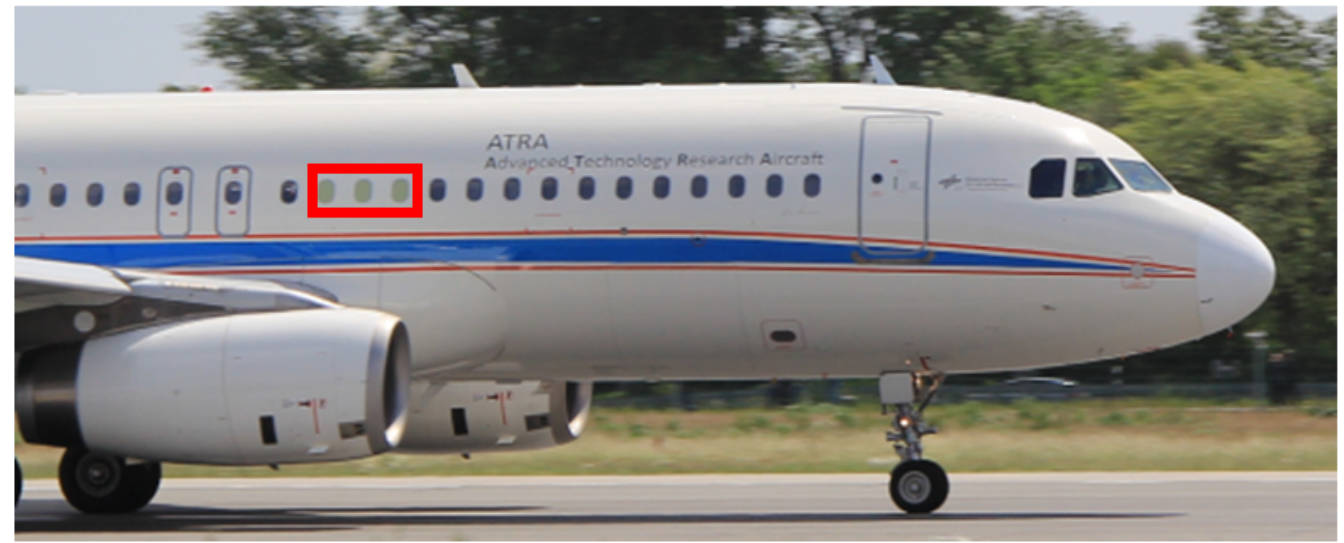

Figure 1. DLR ATRA test carrier at takeoff with pressure transducer array

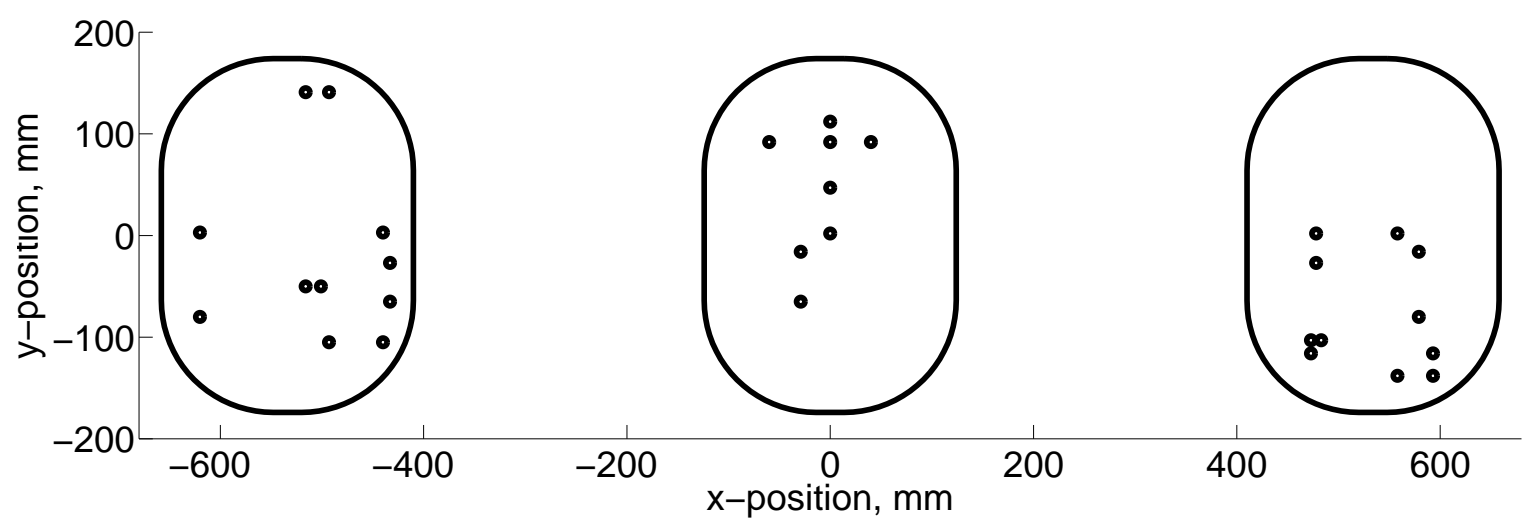

Figure 2. Sketch of dummy windows with Kulite positions on the aircraft starboard side; view from inside

\section{B. Experimental Setup}

The flight condition at a Mach number of $M a=0.78$ at an altitude of $10668 \mathrm{~m}$ (FL350) was used for further analysis, since it provided a continuous measurement duration of $10 \mathrm{~min}$. Boundary layer thickness $\delta$ was determined by an estimation for large Reynolds numbers provided by Bies, ${ }^{17}$ which was adapted to the experimental in-flight boundary layer data of Gyorgyfalvy ${ }^{18}$ at a similar aircraft position on a Boeing model 720 jetliner. The estimation led to a value of $\delta=0.17 \mathrm{~m}$ at the array position.

\section{Data Processing}

\section{A. Cross-Spectral-Density}

The cross-spectral density matrix $R$ of transducer signals yields information about the compliance and magnitude of many signals for a certain frequency. It is determined by calculating the cross-power spectrum for each sensor pairing by complex conjugate multiplication of the Fourier transforms of the time signals

$$
P_{m}(\omega)=\frac{1}{2 \pi} \int_{-\infty}^{+\infty} p_{m}(t) e^{-\mathrm{j} \omega t} \mathrm{~d} t
$$




$$
P_{n}(\omega)=\frac{1}{2 \pi} \int_{-\infty}^{+\infty} p_{n}(t) e^{-\mathrm{j} \omega t} \mathrm{~d} t
$$

with $m, n=1 \ldots N$ and $N$ being the number of transducers in the array.

The cross power is calculated by

$$
R_{m n}(\omega)=P_{m}(\omega) \cdot P_{n}(\omega)^{*}
$$

where $P^{*}$ denotes the complex conjugate of $P . R_{m n}$ is the Fourier transform of the cross correlation function between signals from transducer $m$ and transducer $n$. Since each sensor pairing on the array is separated by a spacial distance in $x$ and $y$-direction, one can write

$$
R(\xi, \eta, \omega)=R_{m n}(\omega)
$$

with $\xi_{m n}=x_{m}-x_{n}$ and $\eta_{m n}=y_{m}-y_{n}$.

\section{B. Coherence}

Coherence was calculated by normalizing the absolute value of each cross power with the auto power at each transducer position (equation (5)). $\xi$ and $\eta$ will be used as terms for the longitudinal and lateral directions, respectively. It provides standardized information about the linear correlation of the signals at the two positions. A value of $\Gamma=1$ denotes perfect coherence of the two signals, a value of $\Gamma=0$ denotes no similarities between the two signals.

$$
\begin{gathered}
\Gamma_{m n}(\omega)=\frac{\left|R_{m n}(\omega)\right|}{\sqrt{R_{m m}(\omega) R_{n n}(\omega)}} \\
\Gamma(\xi, \eta, \omega)=\Gamma_{m n}(\omega)
\end{gathered}
$$

The expressions on the left hand side of equations (4) and (6) will be used subsequently when referring to the cross-spectral matrix $R$ and the coherence matrix $\Gamma$.

\section{Wavenumber Spectra}

Two-dimensional wavenumber-frequency-spectra were generated by planar-wave-beamforming as described by Ehrenfried and Koop. ${ }^{5}$ The wavenumber-frequency spectrum $P\left(k_{x}, k_{y}, \omega\right)$ can be calculated efficiently for each frequency $\omega$ by taking the spatial Fourier transform of the cross-spectral density matrix $R$.

$$
P\left(k_{x}, k_{y}, \omega\right)=\frac{1}{(2 \pi)^{2}} \int_{-\infty}^{\infty} \int_{-\infty}^{\infty} R(\xi, \eta, \omega) e^{-\mathrm{j}\left(k_{x} \xi+k_{y} \eta\right)} \mathrm{d} \eta \mathrm{d} \xi
$$

When plotting the spectrum, it is convenient to normalize the wavevector-coordinates by the wavenumber $k_{0}$ of a wave with evaluation frequency $f$ propagating at the speed of sound present at the flight level altitude $c=\sqrt{\kappa \cdot \Re \cdot T(F L)}$.

$$
k_{0}=\frac{2 \cdot \pi \cdot f}{c}
$$

\section{Deconvolution}

The wavenumber-frequency-spectrum (WFS) computed from experimental data from the array in figure 2 is initially convolved with the point-spread-function (PSF) shown in figure 3. In order to obtain the desired map with all its significant sources, this "dirty map" from the experiment has to be deconvolved from the array influence. The PSF can be determined when wave propagation models are used to simulate the mapping of a single source. This PSF is used in an algorithm to separate the source-map from the influence of the array. An algorithm for the separation of the PSF from the dirty map in acoustic arrays has been introduced by Brooks $^{19}$ (DAMAS) and was modified by Dougherty ${ }^{20}$ (DAMAS2). A summary and comparison of several iterative deconvolution procedures is given by Ehrenfried and Koop, ${ }^{16}$ where the following methods are explained further. In the following investigation, the DAMAS2 algorithm will be applied to the data. 


\section{DAMAS2}

The deconvolution problem can be described as a system of equations

$$
b=C \cdot q .
$$

In equation (9), $b$ denotes the elements of the dirty map in a column vector, $C$ denotes the matrix representing the PSFs for each source position and $q$ denotes the source map in column vector notation. In contrast to the original paper by Dougherty, here the PSFs in $C$ are not modeled by a spherical wave, but by a planar wave in the two-dimensional wavevector domain

$$
e=\exp \left(\mathrm{j}\left(k_{x} \cdot x+k_{y} \cdot y\right)\right)
$$

Equation (9) is solved iteratively and a non-negativity-constraint is applied to $q$ after each iteration. In order to reduce the computational effort in the solution of the system of equations in equation (9), Dougherty ${ }^{20}$ calculated the solution of the problem in equation (9) via frequency domain convolution of the source vector $q$ with a representative point spread function i.e. a single line of $C$. Although this approach decreases appreciably the time needed to calculate the source map, it only works for deconvolution problems, where the point-spread function of the array can be considered shift-invariant. As described by Dougherty, ${ }^{20}$ a shift-invariant point-spread-function "is such that translating a point source causes the entire beamform map to shift the same amount without significantly changing in any other way". The expression for this is given by

$$
\operatorname{PSF}\left(\vec{x}_{\mathrm{A}}, \vec{x}_{\mathrm{q}}\right)=\operatorname{PSF}\left(\vec{x}_{\mathrm{A}}-\vec{x}_{\mathrm{q}}\right)
$$

where $\vec{x}_{\mathrm{A}}$ is the position of the array and $\vec{x}_{\mathrm{q}}$ is the position of the source.

For planar wave beamforming in the $k_{x}-k_{y}$-domain, this calculation is valid over the entire steering hemisphere. ${ }^{20}$ An exemplary point spread function of the array resulting from excitation by a planar wave at $k_{x} / k_{0}=1$ and $k_{y} / k_{0}=0$ is shown in figure 3 .

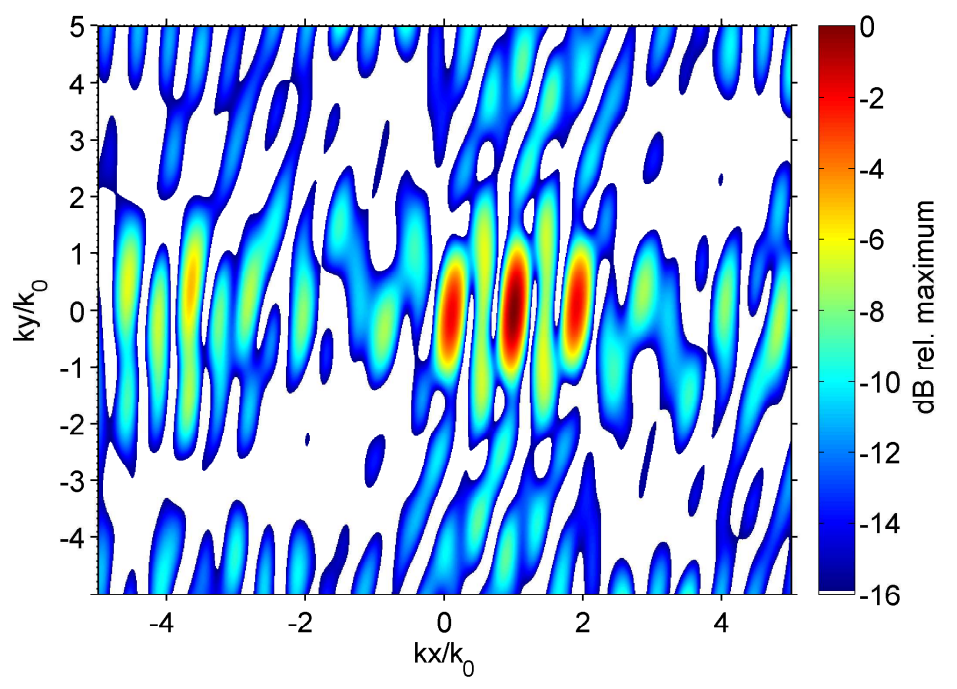

Figure 3. Point-Spread-Function of the Array with a source at $k_{x}=k_{0} ; k_{y}=0$

\section{E. Flow direction}

Flow direction was extracted from the coherence maps via singular value decomposition (SVD) in $\xi, \eta$-space.

For each evaluation frequency of $R$, coherence maps were calculated for all available transducer spacings and interpolated on a mesh in $(\xi, \eta)$-space. A threshold of $\Gamma_{\text {thresh }}=0.05$ was used to extract the shape of the coherent area. Singular value decomposition was utilized in order to find information about orientation and size of the eigenvectors of this area. Subsequently, information about the magnitude ratio of lateral to longitudinal coherence was calculated from the ratio of the eigenvalues. The input vector $D$ for the analysis 
contained the coordinates of the input points in $\xi, \eta$-space, which were obtained by the application of the threshold.

$$
D=\left(\begin{array}{cc}
\xi_{1} & \eta_{1} \\
\xi_{2} & \eta_{2} \\
\vdots & \vdots
\end{array}\right) .
$$

The positive square roots of the eigenvalues $\lambda_{i}$ of the matrix $D^{T} D$ are the standard deviations of the input values multiplied by $\sqrt{N-1}$, where $N$ represents the number of input elements for the analysis. ${ }^{21}$ Therefore, the standard deviation $\sigma_{i}$ of the $i^{\text {th }}$ component can be calculated by

$$
\sigma_{i}=\frac{\sqrt{\lambda_{i}}}{\sqrt{N-1}}
$$

The standard deviation of the SVD in longitudinal direction represents a term analogous to the life-length of a vortex structure as described by Palumbo. ${ }^{8,9}$ Likewise, a factor is used to stretch the eigenvalue to the desired coherence- or life-length (equation (14)).

$$
l_{\max , i}=\sqrt{\pi} \cdot \sigma_{i}
$$

When the flow direction is presumed to coincide with the orientation of maximum spatial coherence, information about the flow angle is accessible. The orientation of the first principal component therefore provides the flow direction.

\section{F. Peak removal}

Distinct $400 \mathrm{~Hz}$ electric tonal noise was present in the Kulite data. In order to suppress the effect of this disturbance while still preserving the information in the characteristic peaks, apart from those arising from electric noise, linear interpolation was applied at multiples of $400 \mathrm{~Hz}$ in order to flatten the spectra.

\section{G. Coherence and Wavenumber Models}

\section{Corcos}

The Corcos model ${ }^{14}$ models the coherence of pressure fluctuations by using coefficients $A\left(\omega \xi / u_{\mathrm{c}}\right)$ and $B\left(\omega \eta / u_{\mathrm{c}}\right)$ for the magnitude. Corcos presents these empirical coefficients based on data by Willmarth and Wooldridge. ${ }^{4}$ Their measurements in the wind tunnel were conducted at a speed of $62 \mathrm{~m} / \mathrm{s}(M a \approx 0.18)$ and the boundary layer was estimated to have a thickness of $\delta \approx 127 \mathrm{~mm}$. In the paper, the usage of exponential functions for the calculation of $A$ and $B$ is proposed.

$$
A\left(\omega \xi / u_{\mathrm{c}}\right)=\exp \left(\frac{|\xi|}{\Omega_{\xi}}\right) \quad ; \quad B\left(\omega \eta / u_{\mathrm{c}}\right)=\exp \left(\frac{|\eta|}{\Omega_{\eta}}\right) .
$$

The decay factors for the exponential is given in the literature and can also be obtained by fitting an exponential function through the data provided by Corcos. By using exponential functions for the decay of the coherence, this model can be easily transformed into wavenumber space using equation (7). The transformation yields equation (17).

$$
\begin{aligned}
\Gamma(\xi, \eta, \omega) & =A\left(\frac{\omega \xi}{u_{\mathrm{c}}}\right) B\left(\frac{\omega \xi}{u_{\mathrm{c}}}\right) \exp \left(\frac{-\mathrm{j} \omega \xi}{u_{\mathrm{c}}}\right) \\
P_{\text {corc }}\left(k_{\xi}, k_{\eta}, \omega\right) & =\frac{4 \Omega_{\xi} \Omega_{\eta}}{\left[1+\left(k_{\xi} \Omega_{\xi}+\frac{\omega \Omega_{\xi}}{u_{\mathrm{c}}}\right)^{2}\right]\left[1+\left(k_{\eta} \Omega_{\eta}\right)^{2}\right]} .
\end{aligned}
$$

Given that the coherent pressure fluctuations are caused by vortex structures of distinct size, the Corcos model does not take into account a maximum possible vortex structure size due to the boundary layer thickness. Thus, large structures, which are considered to be the cause for spatial coherence in the lowfrequency range, are not limited to values below $\delta$. As a result, the coherence length will rise to infinity with decrease of frequency. However, a contrary effect has been observed by many authors. (Bhat, ${ }^{6}$ Farabee, ${ }^{22}$ Palumbo, ${ }^{8}$ Ehrenfried \& Koop ${ }^{5}$ ) 


\section{Efimtsov}

The Efimtsov model ${ }^{7}$ is similar to the Corcos model, for it, too, models the decay of the coherence by exponential functions. However, the boundary layer thickness is taken into account and serves as a filter for the coherence at low frequency. It was deduced from flight test data at various speeds from $M a=0.41$ up to $M a=2.1$ and a Reynolds Number ranging from $R e_{x}=0.5 \cdot 10^{8}$ up to $R e_{x}=4.85 \cdot 10^{8}$. No information on the exact speeds, the aircraft size, positioning on fuselage, flight altitude, and boundary layer thickness is given. Efimtsov uses a "correlation space scale" $\Lambda\left(\omega, \Delta \mathrm{x}, u_{\mathrm{c}}, \delta, \tau_{w}, \rho\right)$, which scales with boundary layer thickness. It is similar to $L\left(\omega, \Delta \mathrm{x}, u_{\mathrm{c}}\right)$ used by Corcos. The exponential model is described by equation (18). The model transformed into wavenumber space by a spatial Fourier transformation is shown in equation (19). Due to its many input parameters, the Efimtsov model has the potential to provide good agreement with the measured flight test data in the present investigation. However, since this model requires so many input parameters that are rather difficult to determine (as $\tau_{\mathrm{w}}$ and $\rho$ ), its application is complicated and depends on assumptions for their values, especially for the cases where they cannot be measured.

$$
\begin{gathered}
\Gamma(\xi, \eta, \omega)=\exp \left(-\frac{|\xi|}{\Lambda_{\xi}}-\frac{|\eta|}{\Lambda_{\eta}}-\frac{j \omega \xi}{u_{\mathrm{c}}}\right) \\
P_{\text {efim }}\left(k_{\xi}, k_{\eta}, \omega\right)=\frac{\Lambda_{\xi} \Lambda_{\eta}}{\pi^{2}\left[1+\left(k_{\xi} \Lambda_{\xi}+\frac{\omega \Lambda_{\xi}}{u_{\mathrm{c}}}\right)^{2}\right]\left[1+\left(k_{\eta} \Lambda_{\eta}\right)^{2}\right]}
\end{gathered}
$$

\section{Results}

\section{A. Coherence}

Coherence was calculated for each transducer spacing and evaluation frequency. Results from SVD are shown in figure 4 with transducer spacings used for SVD analysis. At all the spacings used a coherence value larger than threshold was present. The eigenvector resulting from SVD analysis having the largest eigenvalue was considered as longitudinal flow direction. It is shown in the figure as a solid black line, whereas the perpendicular, or lateral direction is depicted as a dashed black line.

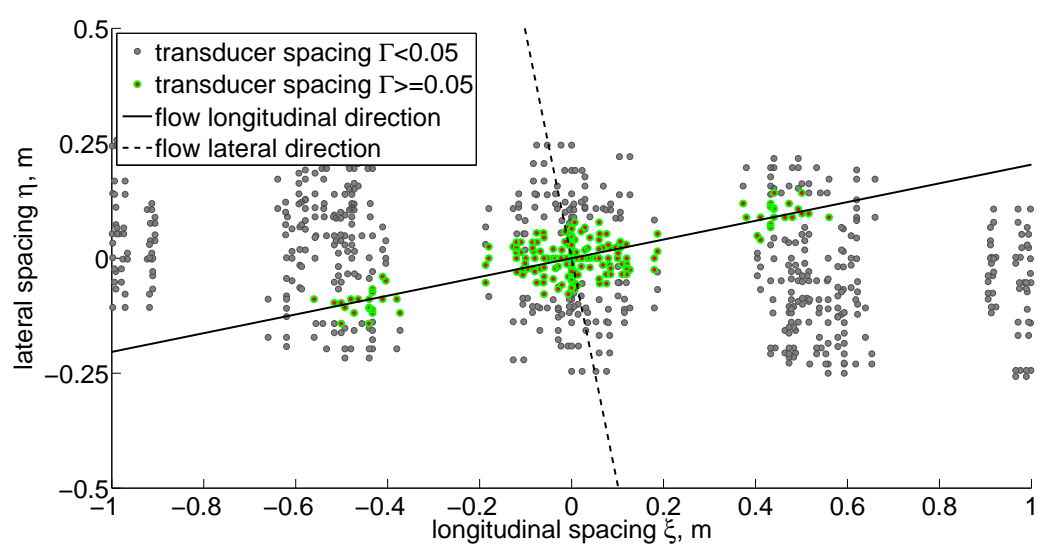

Figure 4. Transducer spacings used for flow direction analysis at $f=1349 \mathrm{~Hz}, \mathrm{Ma}=0.78$, FL350

In figure 5, coherence is depicted two-dimensionally in $\xi, \eta$-space for a frequency of $1349 \mathrm{~Hz}$. This analysis frequency relates to a wave number at the speed of sound of $k_{0}=28.58 \mathrm{~m}^{-1}$. As expected, coherence reaches a value of 1 at a spacing of $\xi, \eta=0$ and is point-symmetric around the origin. Regular oscillations in the coherence as observed by Arguillat ${ }^{15}$ in the low-speed range can be seen in the two-dimensional plot in stream-wise direction in the vicinity of the origin. These oscillations extend over a distance of approximately $0.2 \mathrm{~m}$.

One-dimensional coherence, corresponding to a slice through the interpolated coherence in figure 5 , is shown in figures 7 ( $\xi$ linear) and 6 ( $\xi$ logarithmic) for two directions: first the direction of maximum coherence length ("flow" direction) and second aircraft longitudinal direction ("a/c" direction). The principle of this is 


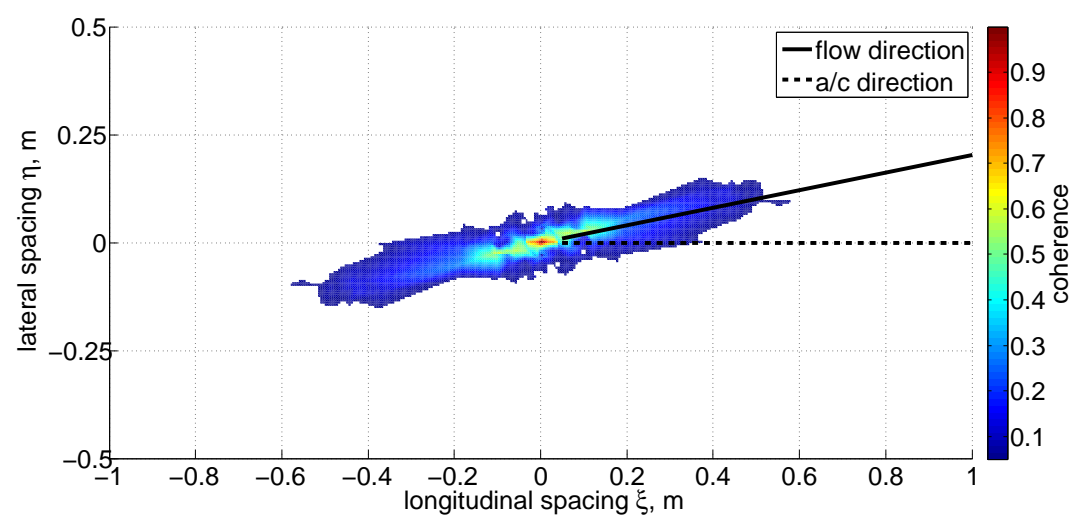

Figure 5. 2D-Coherence extracted from flight test at $f=1349 \mathrm{~Hz}, M a=0.78, F L 350$

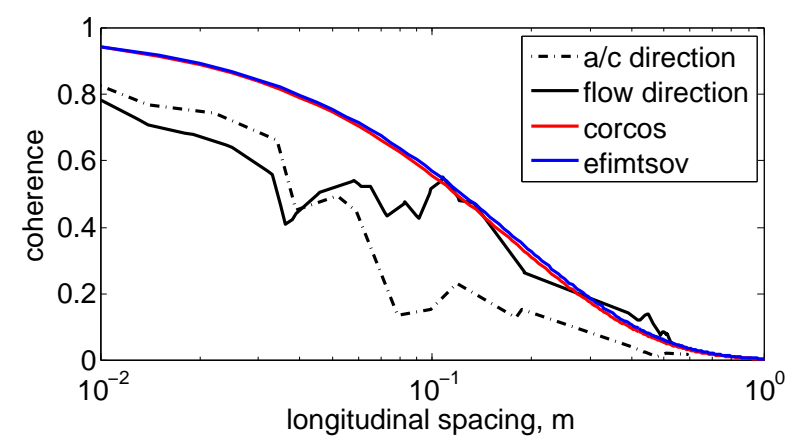

Figure 6. Coherence slices in flow direction and a/c longitudinal direction with coherence models (logarithmic scale)

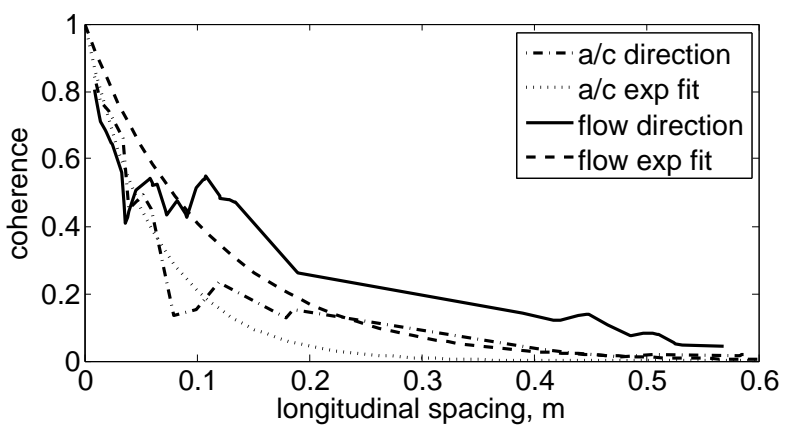

Figure 7. Coherence slices in flow direction and a/c longitudinal direction with exponential fits (linear scale)

illustrated in figure 5. Coherence in flow direction exceeds the coherence in aircraft longitudinal direction, as can be seen in figure 6 . In the spacing region $\xi<5 \mathrm{~cm}$ however, the level of coherence is higher for the a/c direction than for the flow direction (except for $\xi=0$, where a coherence value of $\Gamma(0,0, \omega)=1$ is present).

Both the experimental data and the predictions from the models of Corcos and Efimtsov are depicted in this figure. Both models show a good agreement with the values in flow direction at spacings $\xi>0.2 \mathrm{~m}$. Below this value, coherence levels are overestimated by the models. In aircraft longitudinal direction, coherence levels are below the values predicted by the models for all spacings considered.

In order to compare the experimental data with the Corcos and the Efimtsov model, exponential fits with an ansatz function $\Gamma(\xi)=\exp (L \cdot \xi)$ are applied to the data and shown in figure 7 . When fitting the data in flow direction, the points at great spacings are not well represented by the shape of an exponential function. The fit shows good agreement in the spacing area $\xi<0.2 \mathrm{~m}$. Coherence values at greater spacings are not well matched. An exponential decay factor of $L_{\text {flow }}=-15.57$ is obtained for the evaluation in flow direction, while a value of $L_{\mathrm{a} / \mathrm{c}}=-8.85$ is obtained from the evaluation in aircraft longitudinal direction. This accounts for a deviation of $\frac{L_{\mathrm{flow}}-L_{\mathrm{a} / \mathrm{c}}}{L_{\mathrm{flow}}}=43 \%$ in the exponential coefficients when the flow direction is not taken into account.

A different approach of the ansatz function is shown in figure 8. Here, the drop in coherence is described as subsequent decay processes:

$$
\Gamma(\xi)=L_{1}(\xi) \cdot e^{L_{2}(\xi) \cdot \xi} .
$$

The first decay occurs at spacing values of $\xi \leq \xi_{\text {thresh }}$ whereas the second collapse appears at spacing values greater than the threshold. The double fit accounts for both the requirement of $\Gamma=1$ at $\xi=0$ and the steady drop at large spacings. For a first glance of the goodness of this fit, $\xi_{\text {thresh }}=0.02 \mathrm{~m}$ was used 


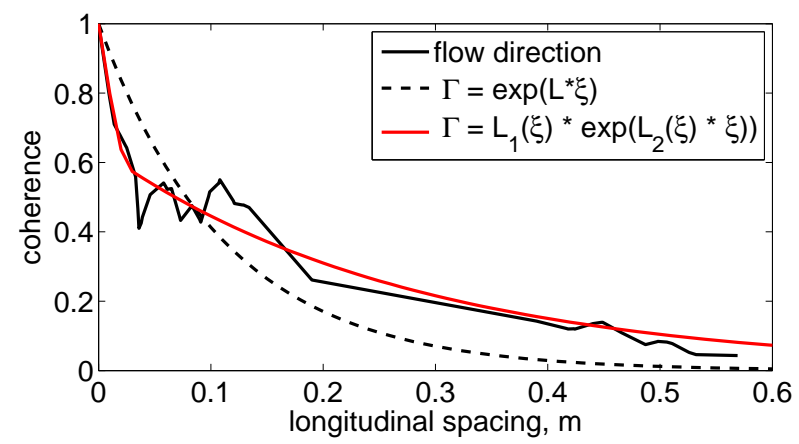

Figure 8. Coherence slice in flow direction and double exponential fit

and the fit was applied to the flow direction coherence data. The fit parameters are described in table 1. The suitability of the fit gives reason for the assumption that the decay process in the boundary layer under examination is a twofold process. However, further examination of this effect is needed to draw reliable conclusions about appearance and characteristic.

\begin{tabular}{|c|c|c|}
\hline & $L_{1}$ & $L_{2}$ \\
\hline$\xi \leq \xi_{\text {thresh }}$ & 1 & -22.54 \\
\hline$\xi>\xi_{\text {thresh }}$ & 0.64 & -9.68 \\
\hline
\end{tabular}

Table 1. Parameters for the double exponential fit

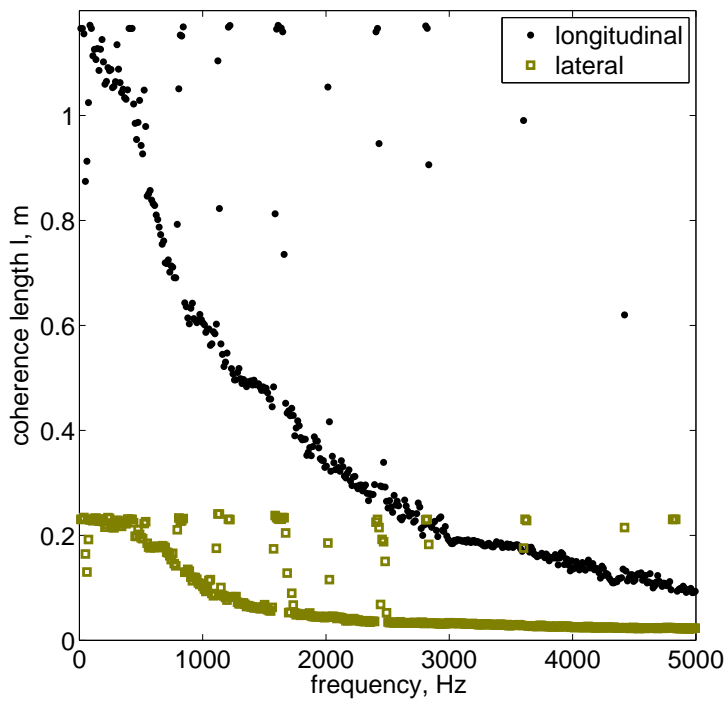

Figure 9. Coherence length extracted from flight test

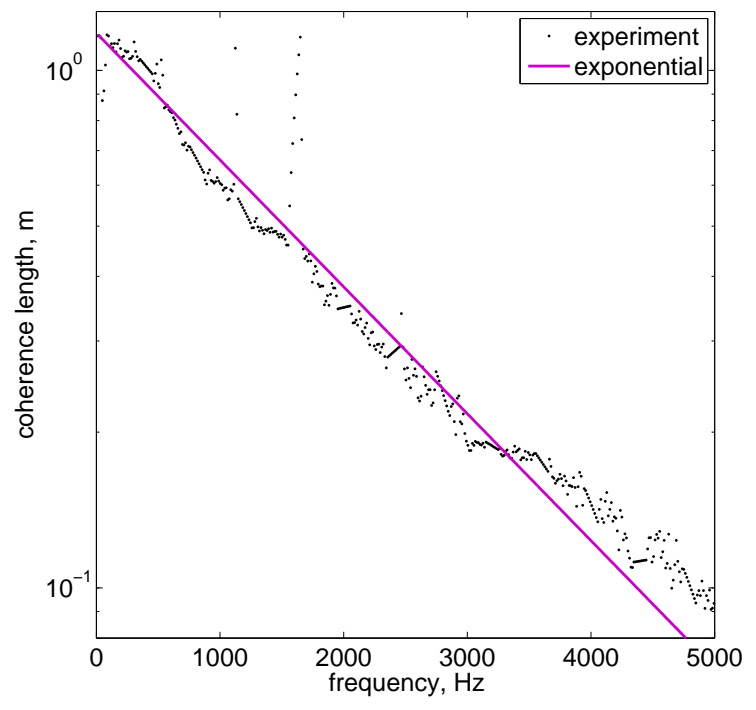

Figure 10. Coherence length fits data

Coherence lengths as calculated in equation (14) are shown in figure 9 for different frequencies. Both longitudinal and lateral coherence lengths show a drop with increasing frequency above $500 \mathrm{~Hz}$. In the low-frequency range, a maximum at approximately $100 \mathrm{~Hz}$ can be observed. A distinct peak is visible at $f=1618 \mathrm{~Hz}$ in lateral direction (and less pronounced, in longitudinal direction). This tone is caused by the blade passage in the jet engine fan.

An exponential fit through the longitudinal coherence length data is shown in figure 10. The peaks caused by the electrical noise were removed before fitting. The the exponential shape shows a good agreement with 


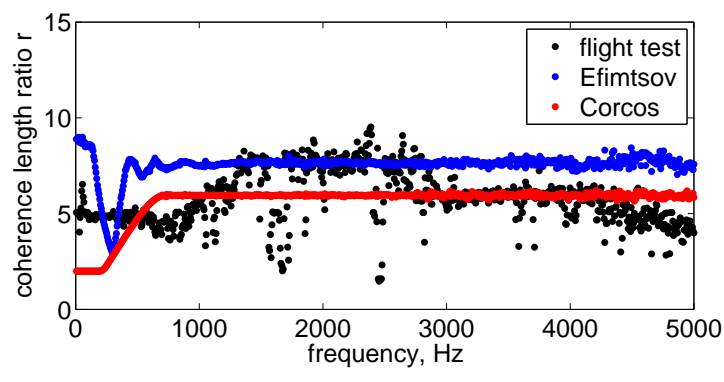

Figure 11. Ratio of longitudinal to lateral coherence

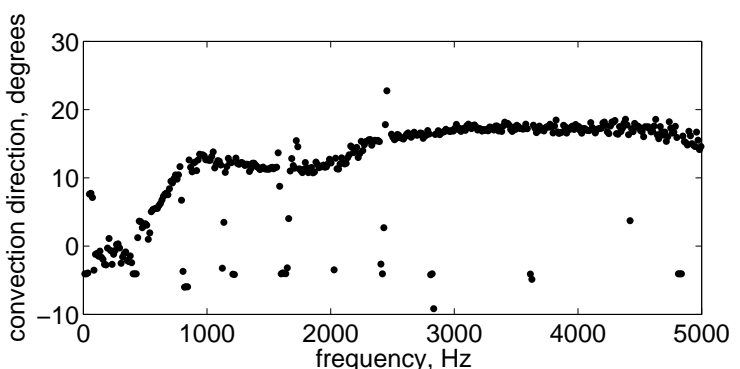

Figure 12. Angle of maximum coherence length

the experimental data.

The length in longitudinal direction exceeds the length in lateral direction by a ratio depending on frequency. It is depicted in figure 11. This ratio $r$ varies from a minimum value of $r \approx 5$ at high and low frequencies to a value of approximately $r \approx 8$ in a frequency range from $f=1400 \mathrm{~Hz}$ until $2800 \mathrm{~Hz}$. The Coherence exhibits a level above threshold throughout the whole spacing area in the low-frequency range. Therefore, the ratio extracted by analysis approaches the ratio of maximum longitudinal to lateral spacings. Again, peaks at multiples of $400 \mathrm{~Hz}$, as described beforehand, are visible in the graph.

The ratios of the models of Efimtsov and Corcos are shown in figure 11 for comparison. These ratios were generated by modeling the coherence with both models two-dimensionally and applying the same analysis as for the experimental data. The Efimtsov model shows a good ratio agreement from $1400 \mathrm{~Hz}$ until $3000 \mathrm{~Hz}$ Above this frequency, the ratio resulting from Corcos model provides the better fit to the experimental data.

Both, the Corcos and Efimtsov- models show a discrepancy in the low-frequency range. For the Corcosmodel, this difference is caused by the analysis, which does not take account of the limitations of vortex structure size due to the boundary layer thickness. Therefore, the values for both longitudinal and lateral coherence lengths increase asymptotically to the borders of the spacing area chosen for analysis.

The Efimtsov model considers these vortex size limitations. However, the correlation space scale $\Lambda$ does not change equally in lateral and longitudinal direction to compensate for the limitation. For this reason, and also because of the limited resolution in the analysis area, an oscillation occurs as seen in the low-frequency part in figure 11.

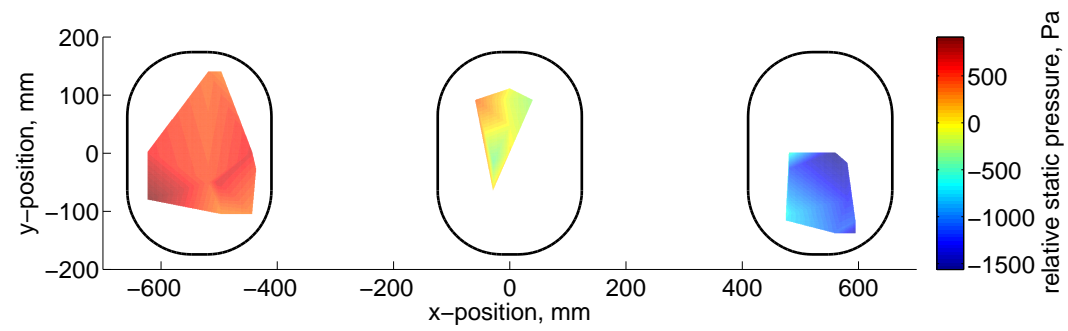

Figure 13. Relative static pressure distribution over the array

The angle of the eigenvector of the first component extracted from SVD relative to the aircraft longitudinal axis is depicted in figure 12. Starting at a downward angle of $\alpha \approx-2^{\circ}$ a continuous rise of the value can be observed until $f \approx 900 \mathrm{~Hz}$, where a direction of $\alpha \approx 12^{\circ}$ upwards is predominant. From $f=2000 \mathrm{~Hz}$ until $f=3000 \mathrm{~Hz}$ the angle rises to a value of $\alpha \approx 17^{\circ}$. At higher frequencies, direction drops slightly to a level of $\alpha \approx 15^{\circ}$. The downwards angle in the low-frequency domain is caused by the coherence level exceeding the threshold at all spacings. Therefore, all the transducer spacings are considered for the analysis and the orientation of the spacings is the result of the analysis. Apart from this, the extracted flow angle is significantly greater than for a typical angle-of-attack in a cruise condition. The local surface flow does not necessarily resemble the overall flow direction in flight due to the wing pressure field and unknown surface pressure gradients as described by Gyorgyfalvy. ${ }^{18}$ The pressure field is depicted in figure 13 and shows a decrease of static pressure in the lower right position of the array. This is the part of the array closest to 
the wing.

At the threshold of $\Gamma_{\text {thresh }}=0.05$ used, maximum coherence length was found to be $l_{\max }=0.51 \mathrm{~m}$ at an upward angle of $\alpha_{\max }=12^{\circ}$ relative to the aircraft longitudinal axis (figure 12).

Simulated 2D-coherences were calculated applying the same flight conditions of $M a=0.78$ at FL350 to the models of Corcos and Efimtsov. For the Efimtsov model, a friction coefficient $c_{f}$ determined by Gyorgyfalvy ${ }^{18}$ at a similar aircraft length position was used to calculate the wall shear rate $u_{\tau}$. Results from both models are shown in figures 14 and 15. A flow angle $\alpha_{\max }$ determined from experimental data was applied to the graphs for better comparison. Good general agreement can be seen between the models and the measured data (figure 5), especially for the model of Efimtsov which has a similar ratio of longitudinal to lateral coherence length at this frequency. The Corcos model provides greater coherence length in lateral flow direction than determined from the experiment at the given frequency.

www

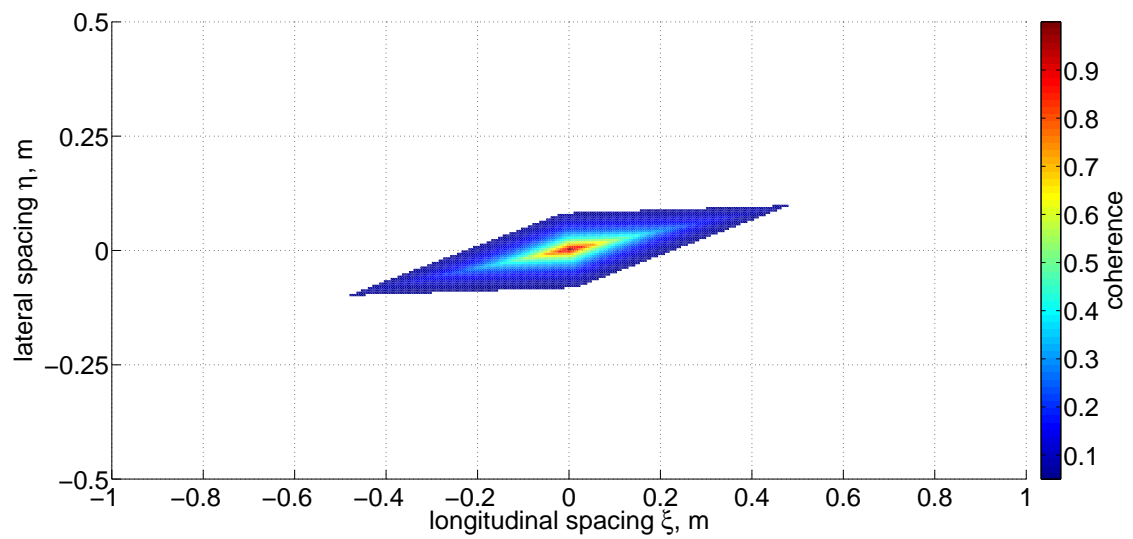

Figure 14. Coherence from Corcos model at $f=1349 \mathrm{~Hz}, M a=0.78, F L 350$

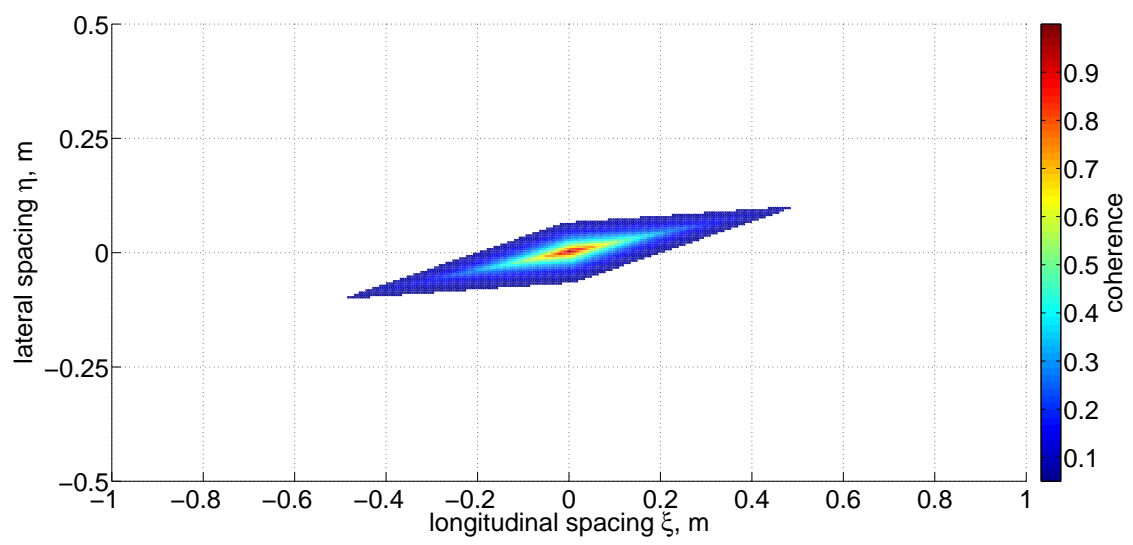

Figure 15. Coherence from Efimtsov model at $f=1349 \mathrm{~Hz}, M a=0.78, F L 350$ 


\section{B. Wavenumber Spectra}

The wavenumber-frequency-spectrum (WFS) is a depiction of the planar waves that strike the array from different directions. Using a WFS, an illustration of convectively transported pressure oscillations over the array is possible.

A WFS is calculated for each frequency by performing a spatial Fourier Transform on the cross spectral density matrix $R$. An exemplary result ("dirty map") at a frequency of $f=1349 \mathrm{~Hz}$ calculated with equation (7) is given in figure 16. The convective ridge is visible as an elongated region of high amplitude in the right half of the spectrum. However, the influence of the array's PSF is noticeable as a significant widening of the ridge and a considerably higher surrounding level. After applying a deconvolution to the dirty map, the approximate underlying source map becomes visible (figure 17). The convective ridge emerges clearly in the spectrum. The inclination of the convective ridge and the angle its center is shifted towards the $k_{x}$-axis are indicators of the predominant flow direction. Lateral branches of the convective ridge in $k_{y}$-direction extend up to $4 \cdot k_{0}$. In the $k_{x}$-direction, statements about the extension are difficult, since remnants of the PSF are still present left and right of the ridge. The fact that the deconvolution procedure did not remove all relicts from the PSF is believed to be caused by a pressure gradient predominant in the area of the transducer array (figure 13). The pressure over the array is shown relative to a sensor positioned in the middle of the center dummy window. The PSF is believed to be altered slightly in a way that does not comply completely with the wave propagation model used. A source visible in the acoustic domain at $\left(k_{x}, k_{y}\right) \approx(0.7,0)$ cannot yet be assigned to either an actual acoustic source present in the flight, or to an artifact from the analysis.

The dotted line in figure 17 encircles the acoustic region defined by

$$
\left(\frac{k_{x}}{k_{0}}, \frac{k_{y}}{k_{0}}\right)=\left(\frac{\cos \theta}{1+M a \cos \theta}, \frac{\sin \theta}{1+M a \cos \theta}\right) .
$$

All waves propagating at the speed of sound at a direction parallel to the array surface are mapped onto the boundary of the ellipse. Waves striking the array in a non-parallel manner are mapped inside the ellipse, thus appearing to have a phase velocity greater than $c$. The alignment of the acoustical domain was adapted to the center of the convective ridge described below.

Using the spectrum, information about narrow-band convection velocity is accessible. The alignment of the convective ridge is extracted by adopting a threshold to the data around the maximum value and applying a singular value decomposition to the wave number coordinates above the threshold. The intersection point of the two principle components is taken as center point for the convective ridge. This point yields the magnitude of the convective velocity $u_{\mathrm{c}}$.

$$
\begin{gathered}
u_{\mathrm{c}}=\frac{2 \pi \omega}{k_{\max }} \\
k_{\max }=\sqrt{k_{x, \text { max }}^{2}+k_{y, \text { max }}^{2}}
\end{gathered}
$$

The center of the convective ridge in figure 17 is located at a wavenumber of $k_{\max }=44.4 \mathrm{~m}^{-1}$ from the origin of the spectrum. This represents a convective velocity ratio of $u_{\mathrm{c}} / u_{0} \approx 0.83$ when using the free-stream velocity for normalization.

When evaluating the convective ridge for each frequency, narrow band information about the convection velocity magnitude $u_{\mathrm{c}}$ can be extracted (figure 18). The velocity shows a very steep inclination in the low-frequency range from zero to $475 \mathrm{~Hz}$ where a maximum normalized value of $u_{\mathrm{c}} / u_{0} \approx 0.9$ is reached. Above $475 \mathrm{~Hz}$ the convection velocity decreases linearly with increasing frequency to reach a value of 0.75 at $f=5000 \mathrm{~Hz}$. The convection velocities were extracted from the dirty map. The magnitude of the normalized convection velocity is comparable with that observed by Bhat in flight tests on a Boeing 737 model in his wide-band analysis for spacings of $\xi / \delta=0.65$. Farabee ${ }^{22}$ observed convection velocities with lower magnitudes from measurements in wind tunnel at low speeds, even at a greater value of $\xi / \delta=5.0$.

A peak value of convection velocity observed by Farabee to occur at a Strouhal number of $S h_{\max } \approx 50$ in the wind tunnel is observed to occur at $S h_{\max } w w w . \approx 84$ at this flight condition (Bhat: ${ }^{6} S h_{\max } \approx 143.8$ ). Both the boundary layer thickness $\delta=0.17 \mathrm{~m}$ and the wall shear rate $u_{\tau}=6.08 \mathrm{~m} / \mathrm{s}$ were estimated in this calculation. These are the same values as used for the calculation of the Efimtsov coherence model in section III.A. 


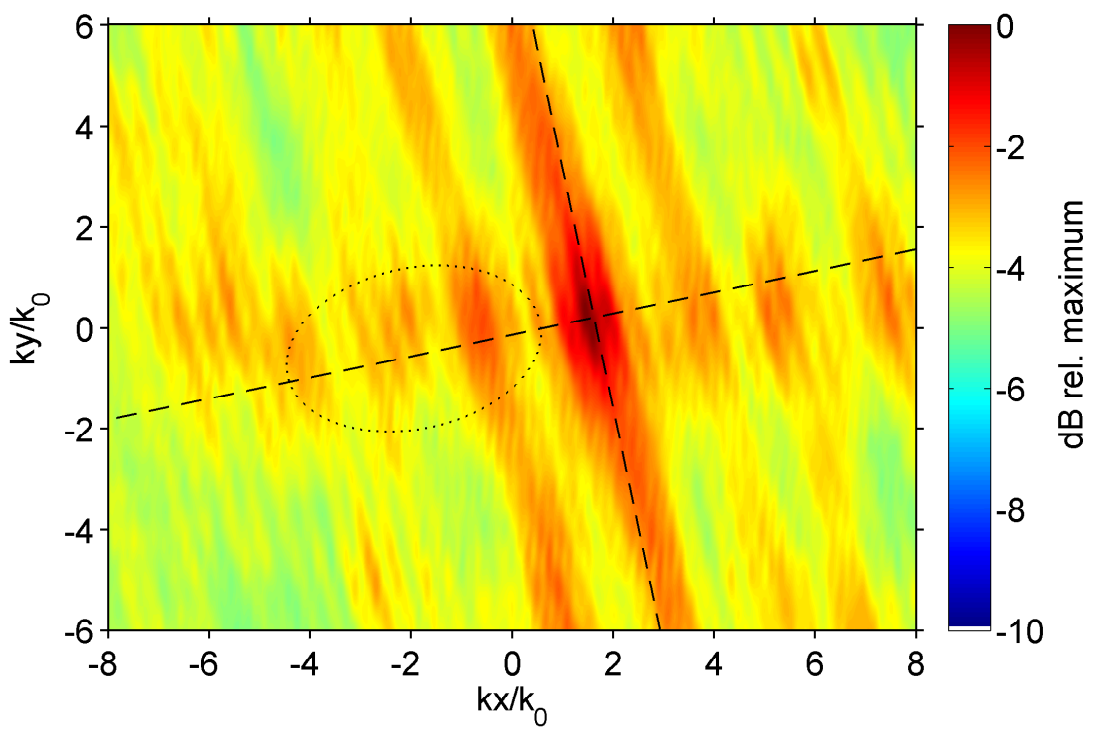

Figure 16. WFS dirty map; $f=1349 \mathrm{~Hz}, M a=0.78, F L 350$

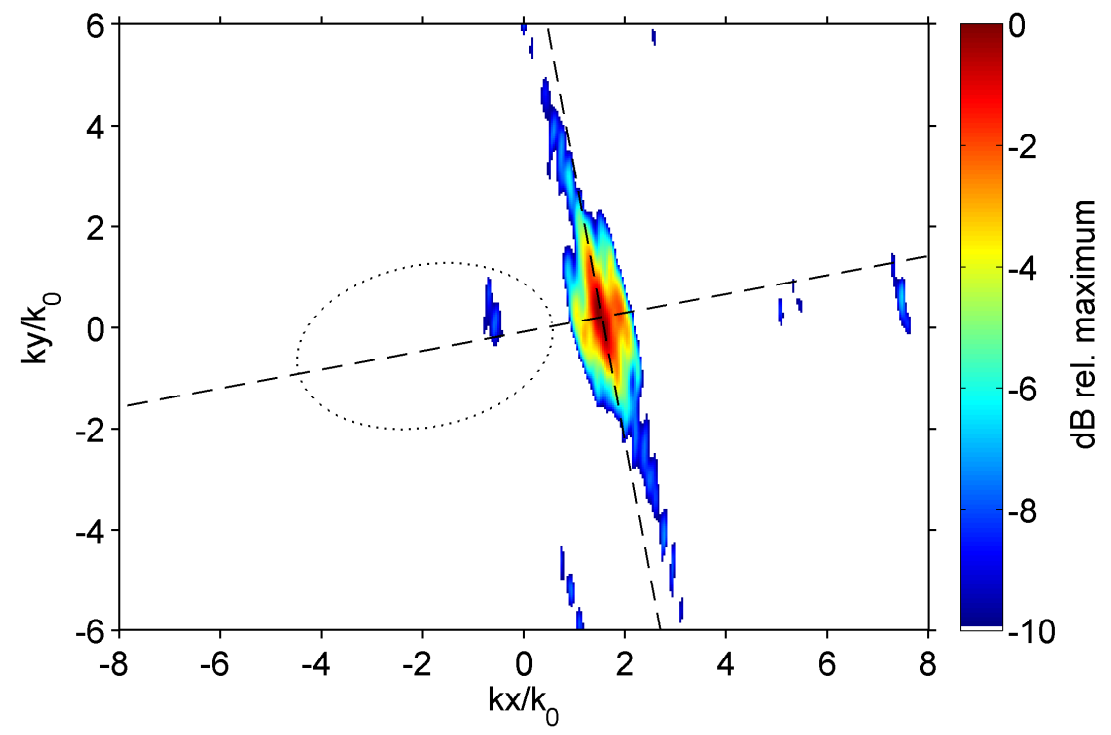

Figure 17. WFS deconvolved with DAMAS2; $f=1349 \mathrm{~Hz}, M a=0.78, F L 350$ 


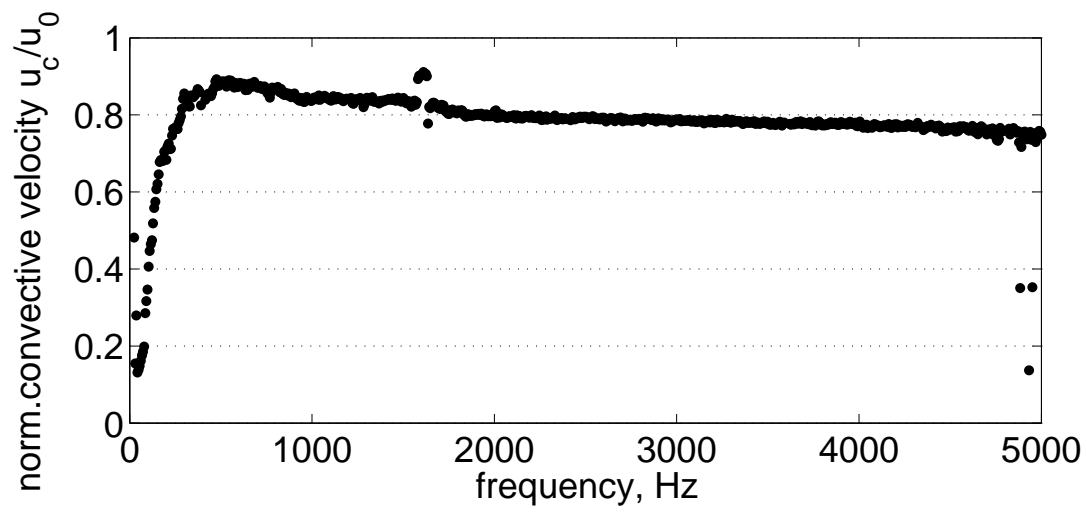

Figure 18. Convective velocity

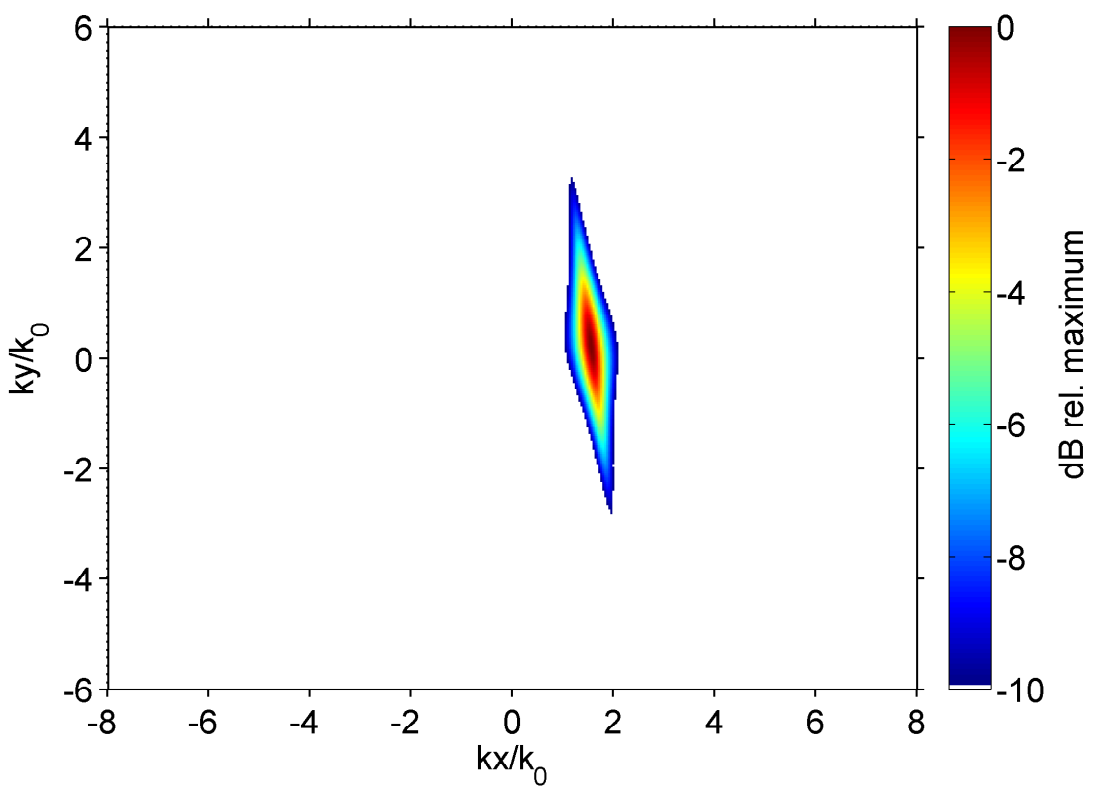

Figure 19. Source map calculated from the Corcos model

When calculating the source maps at a frequency of $f=1618 \mathrm{~Hz}$, influence of the blade-passage frequency of the jet engine becomes visible as a single dominant source in a region above and to the left of the origin (figure 22). This point lies inside the acoustic domain at a value of $k_{y} \approx 1$ and a value of $k_{y} \approx-0.65$. This alludes to the assumption that the predominant wave travels at the speed of sound and strikes the array from a lower aft position.

However, the actual position of the engines approximately vis-à-vis to the microphone array leads to the assumption that the position of the peak in the WFS is to be found at a value of $k_{x} \approx 0$. The shift to the left in the spectrum can be explained by the deformation of the acoustical region due to the free flow above the boundary layer (equation (21)). The propagation velocity component of acoustical waves within the free flow above the boundary layer is delayed in the reverse flow direction. Acoustical waves detected by the array analysis in reverse flow direction therefore appear to have a larger than actuals wave numbers (figure 21)

The maximum amplitude of the convective ridge is approximately $4 \mathrm{~dB}$ lower than the amplitude of the peak of the jet engine at the frequency of occurrence. 


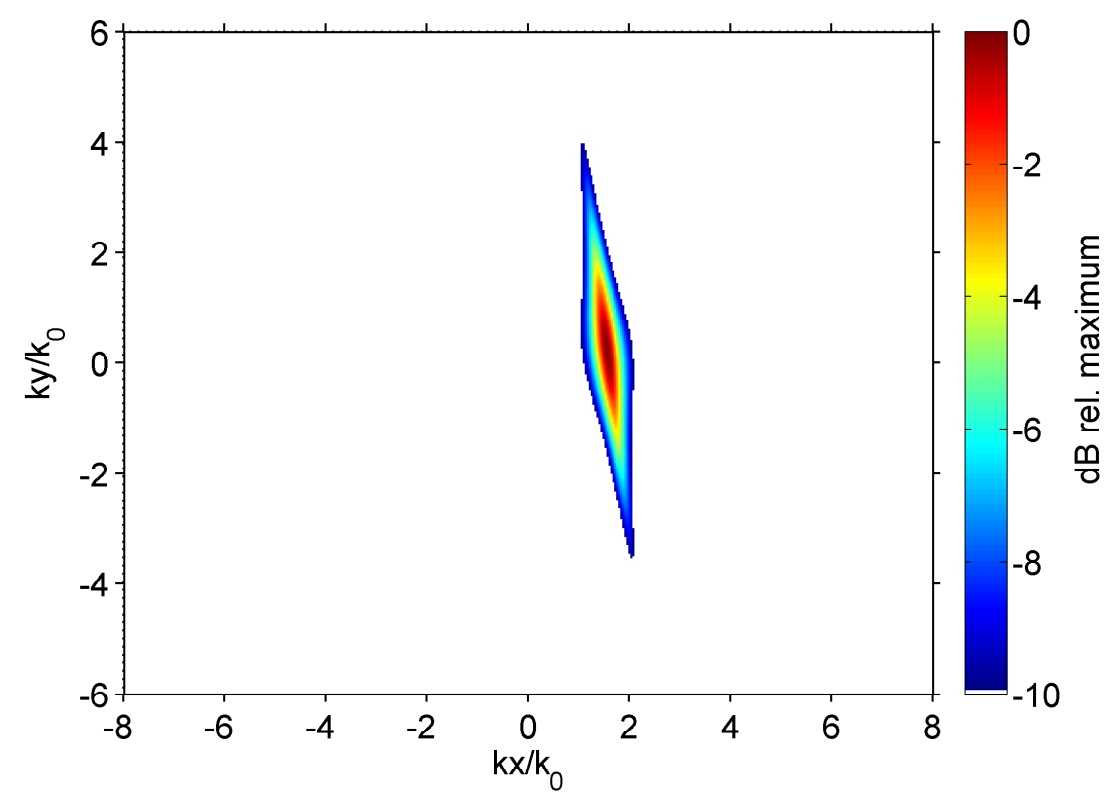

Figure 20. Source map calculated from the Efimtsov model

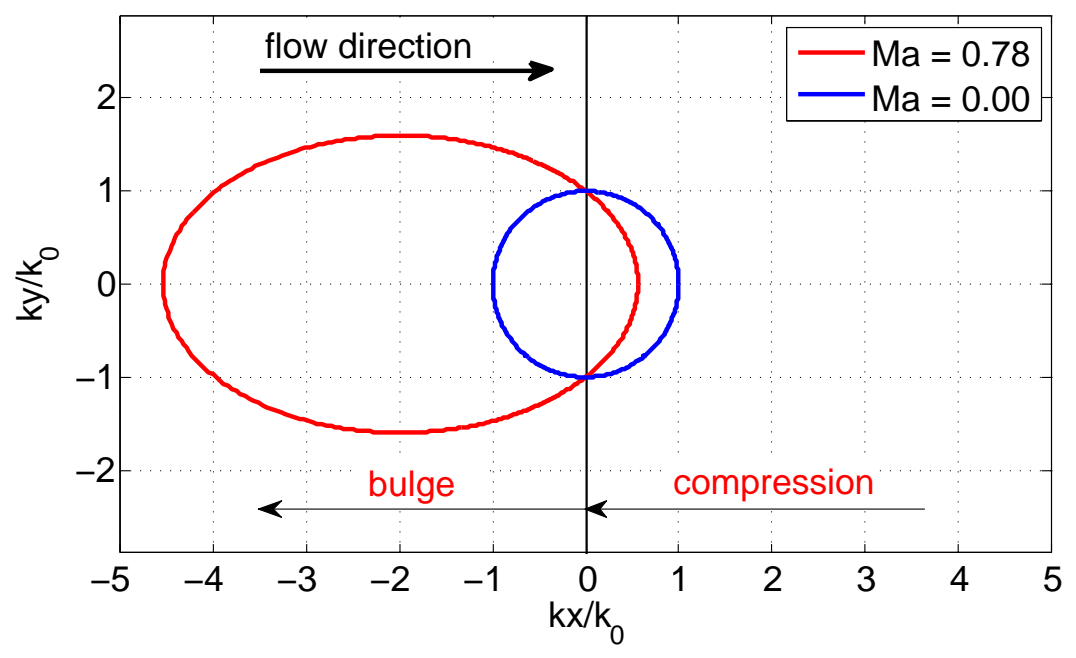

Figure 21. Deformation of the acoustical domain due to flow speed 


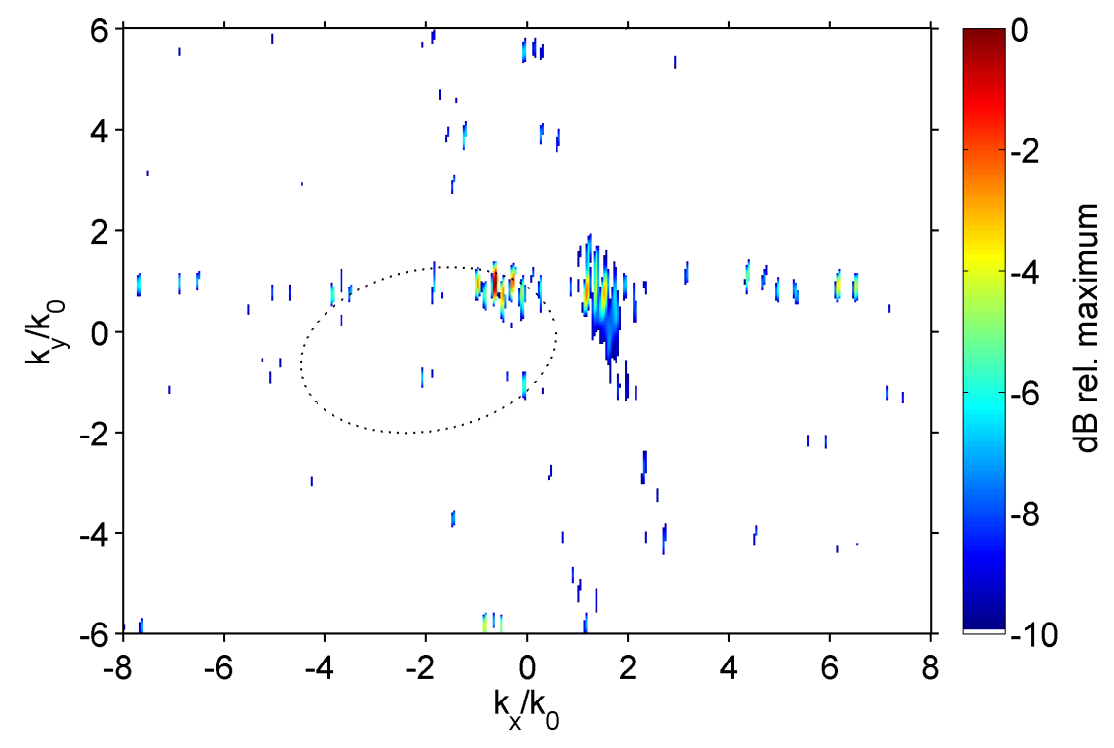

Figure 22. WFS with fan noise visible; $f=1618 \mathrm{~Hz}, M a=0.78$, F L350

\section{Conclusion}

The in-flight applicability of an irregularly-shaped microphone array and the evaluation of the data were illustrated. Data from a flight test was evaluated in order to gain knowledge on convective pressure fluctuations on the fuselage. The data was obtained by using a pressure transducer array installed in three aluminum dummy windows at a position located in the forward wing area. Processing the signals from each sensor pairing yielded two-dimensional coherence plots. The angle of maximum coherence length can be used to obtain information about local flow direction. Comparison of the measured data to the models of Corcos and Efimtsov showed good agreement concerning size and dimension ratios. The quality of the agreement depended on the frequency at which a comparison was made. Coherence decay was determined in flow direction and in aircraft longitudinal direction. Considerable discrepancies were found, when the flow direction does not match the array alignment.

Wavenumber spectra were calculated and deconvolved in the wavenumber domain using an iterative deconvolution scheme. The convective ridge was identified in the spectrum and analyzed to find the convective velocity at each frequency. The characteristic of this convective velocity was found to be similar to that obtained in other studies. The position of its maximum was found to be at a higher Strouhal number than in wind tunnel measurements at low Mach number and at a lower Strouhal number than in other flight test measurements.

A comparison of the deconvolved wavenumber spectra with the models of Corcos and Efimtsov was carried out. At the chosen evaluation frequency, both models show good agreement with the experiment. In the models, less decay from the peak value at the convective wave number towards higher and lower wavenumbers is observed.

The jet-engine fan noise can be identified in the wavenumber spectrum as a distinct peak.

\section{Acknowledgements}

The authors would like to thank the following institutions for their assistance:

- The Federal Ministry of Economics and Technology (BMWi) for the financial support of "SIMKAB" (Simplified Cabin) as part of the aerospace research program (LuFO IV).

- Airbus S.A.S. for the provided infrastructure and help on modifications of the test carrier. 


\section{References}

${ }^{1}$ Mellert, V., Bauman, I., and Freese, N., "Impact of sound and vibration on health, travel comfort and performance of flight attendants and pilots," Aerospace Science and Technology, Vol. 12, 2008, pp. 18-25.

${ }^{2}$ Graham, W., "A Comparison of Models for the Wavenumber-Frequency Spectrum of Turbulent Boundary Layer Pressures," Journal of Sound and Vibration, Vol. 206, No. 4, 1997, pp. 541-565.

${ }^{3}$ Liu, B., "Noise radiation of aircraft panels subjected to boundary layer pressure fluctuations," Journal of Sound and Vibration, Vol. 314, 2008, pp. 693-711.

${ }^{4}$ Willmarth, W. and Wooldridge, C., "Measurements of the fluctuating pressure at the wall beneath a thick turbulent boundary layer," Journal of Fluid Mechanics, Vol. 14, 1962, pp. 187-210.

${ }^{5}$ Ehrenfried, K. and Koop, L., "Experimental Study of Pressure Fluctuations Beneath a Compressible Turbulent Boundary Layer," Paper 2008-2800, 14th AIAA/CEAS Aeroacoustics Conference, Vancouver, British Columbia, AIAA, May 2008.

${ }^{6}$ Bhat, W., "Flight Test Measurement of Exterior Turbulent Boundary Layer Pressure Fluctuations on Boeing Model 737 Airplane," Journal of Sound and Vibration, Vol. 14, No. 3, 1971, pp. 439-457.

${ }^{7}$ Efimtsov, M., "Characteristics of the Field of Turbulent Pressures at the Wall of a Boundary Layer," Sov. Phys. Acoust., Vol. 28, No. 4, 1982, pp. 289-292.

${ }^{8}$ Palumbo, D. L., "Characteristic Lifelength of Coherent Structure in the Turbulent Boundary Layer," Paper 2006-2410, 12th AIAA/CEAS Aeroacoustics Conference, Cambridge, Massachussets, AIAA, May 2006.

${ }^{9}$ Palumbo, D., "Deriving Lifetime Maps in the Time/Frequency Domain of Coherent Structures in the Turbulent Boundary Layer," Tech. Rep. TM-2008-0023921, NASA, 2008.

${ }^{10}$ Rizzi, S. A., Rackl, R. G., and Andrianov, E. V., "Flight Test Measurements From The Tu-144LL Structure/Cabin Noise Experiment," Tech. Rep. TM-2000-209858, NASA, 2000.

${ }^{11}$ Rizzi, S. A., Rackl, R. G., and Andrianov, E. V., "Flight Test Measurements From The Tu-144LL Structure/Cabin Noise Follow-On Experiment," Tech. Rep. TM-2000-209859, NASA, 2000.

${ }^{12}$ Rackl, R. and Weston, A., "Modeling of Turbulent Boundary Layer Surface Pressure Fluctuation Auto and Cross Spectra - Verification and Adjustments Based on TU-144LL Data," Tech. Rep. CR-2005-213938, NASA, 2005.

${ }^{13}$ Spehr, C., Hennings, H., Buchholz, H., Bouhaj, M., Haxter, S., and Hebler, A., "In-flight sound Measurements: A first overview," Submitted for publication, 18th AIAA/CEAS Aeroacoustics Conference, Colorado Springs, Colorado, AIAA, June 2012.

${ }^{14}$ Corcos, G. M., "Resolution of Pressure in Turbulence," The Journal of the Acoustical Society of America, Vol. 35, No. 2, 1963, pp. 192-199.

${ }^{15}$ Arguillat, B., Ricot, D., Robert, G., and C., B., "Measurements of the wavenumber-frequency spectrum of wall pressure fluctuationis uner turbulent flows," Paper 2005-2855, 11th AIAA/CEAS Aeroacoustics Conference, Monterey, California, AIAA, May 2005.

${ }^{16}$ Ehrenfried, K. and Koop, L., "Comparison of iterative deconvolution algorithms for the mapping of acoustic sources," AIAA Journal, Vol. 45, No. 7, 2007, pp. 1584-1595.

${ }^{17}$ Bies, D. A., "A Review of Flight and Wind Tunnel Measurements of boundary Layer Pressure Fluctuations and Induced Structural Response," Tech. Rep. 29134, NASA, 1966.

${ }^{18}$ Gyorgyfalvy, D., "Effect of Pressurization on Airplane Fuselage Drag," Journal of Aircraft, Vol. 2, No. 6, 1965, pp. 531537.

${ }^{19}$ Brooks, T. F., "A Deconvolution Approach for the Mapping of Acoustic Sources (DAMAS) Determined from Phase Microphone Arrays," Paper 2004-2954, 10th AIAA/CEAS Aeroacoustics Converence, Manchester, Great Britain, AIAA, May 2004.

${ }^{20}$ Dougherty, R., "Extension of DAMAS and Benefits and Limitations of Deconvolution in Beamforming," Paper 2005-2961, 11th AIAA/CEAS Aeroacoustics Conference, Monterey, California, AIAA, May 2005.

${ }^{21}$ Bronstein, Semendjajew, Musiol, and Mühlig, Taschenbuch der Mathematik, Verlag Harri Deutsch, 2nd ed., 1995.

${ }^{22}$ Farabee, T. M. and Casarella, M. J., "Spectral features of wall pressure fluctuations beneath turbulent boundary layers," Physics of Fluids A: Fluid Dynamics, Vol. 3, No. 10, 1991, pp. 2410-2420. 